\title{
A New Descriptor Kalman Filter
}

\author{
XU Yan, ZHANG Guosheng
}

Basic Department, Beijing Institute of Graphic Communication, Beijing 102600, P.R.China

E-mail: xuyan@bigc.edu.cn

Keywords: Descriptor systems, Jordan decomposition, Normal systems, Optimal Kalman filtering

\begin{abstract}
Based on Kalman filtering theory in the time domain and Jordan decomposition theory for matrixes, descriptor systems can be changed into normal systems. Then an optimal descriptor Kalman filter is given. Compared with the previous results, it avoids the calculation of ARMA innovation model and white noise estimators. It is a simple and recursive algorithm. It is suitable for real time applications.
\end{abstract}

\section{Introduction}

Descriptor systems are often seen in such fields as circuit, economics and robotics, thus receiving more and more attention. Documents [1 3] present descriptor steady-state Kalman estimators whose drawback is that the optimal estimator is unavailable when observation data are limited. Although the document [4] presents unsteady-state descriptor Kalman estimators, the calculation is huge because the algorithm is based on ARMA model and white noise estimators. In this paper, Jordan decomposition for matrices is used to change a class of descriptor systems into normal systems and Kalman filtering theory is used to introduce a kind of optimal descriptor Kalman filter whose algorithm is recursive and easy for real-time application.

Consider the discrete time descriptor stochastic system

$$
\begin{aligned}
& M x(t+1)=\Phi x(t)+\Gamma w(t), \\
& y(t)=H x(t)+v(t),
\end{aligned}
$$

where the state $x(t) \in R^{n}$, the measurement $y(t) \in R^{m}, w(t) \in R^{r}, v(t) \in R^{m}, M 、 \Phi 、 \Gamma$ and $H$ are the constant matrices.

Assumption 1. The $n \times n$ matrix $M$ is singular, and $\operatorname{rank} M=n-1$.

Assumption 2. $w(t)$ and $v(t)$ are correlated white noises.

$$
\mathrm{E}\left\{\left[\begin{array}{c}
w(t) \\
v(t)
\end{array}\right]\left[\begin{array}{ll}
w^{\mathrm{T}}(j) & v^{\mathrm{T}}(j)
\end{array}\right]\right\}=\left[\begin{array}{cc}
Q & S \\
S^{\mathrm{T}} & R
\end{array}\right] \delta_{i j},
$$

where $\mathrm{E}$ is the expectation, T denotes the transpose, $\delta_{t t}=1, \delta_{t j}=0(t \neq j), S=\mathrm{E}\left[w(t) v^{\mathrm{T}}(t)\right]$ is the correlation matrix.

Assumption 3. The initial state $x(0)$ is independent of $w(t)$ and $v(t)$.

\section{The normalization of a descriptor system}

Lemma 1. If the rank of the $n \times n$ matrix $M$ is $m$, that is $\operatorname{rank} M=m$, then the matrix $M$ has at least $n-m$ zero characteristic root [5,6].

Lemma 2. In the system (1), (2), if the matrix $M$ has $k$ zero characteristic root, then there exists an invertible matrix $F$ such that

$$
M^{\prime} x^{\prime}(t+1)=\Phi^{\prime} x^{\prime}(t)+\Gamma^{\prime} w(t),
$$




$$
y(t)=H^{\prime} x^{\prime}(t)+v(t),
$$

where

$$
\begin{aligned}
& M^{\prime}=F^{-1} M F=\left[\begin{array}{c|c|ccc}
J_{n-k} & & & & \\
\hline & 0 & 1 & & \\
& & \ddots & \ddots & \\
& & & 0 & 1 \\
\hline & & & & 0
\end{array}\right]=\left[\begin{array}{ccc}
J_{n-k} & 0 & 0 \\
0 & 0 & I_{k-1} \\
0 & 0 & 0
\end{array}\right], J_{n-k} \text { is Jordan block, } \\
& \Phi^{\prime}=F^{-1} \Phi F=\left[\begin{array}{ccc|c|cc}
\varphi_{11}^{\prime} & \cdots & \varphi_{1, n-k}^{\prime} & \varphi_{1, n-k+1}^{\prime} & \cdots & \varphi_{1 n}^{\prime} \\
\cdots & \cdots & \cdots & \cdots & \cdots & \cdots \\
\varphi_{n-k, 1}^{\prime} & \cdots & \varphi_{n-k, n-k}^{\prime} & \varphi_{n-k, n-k+1}^{\prime} & \cdots & \varphi_{n-k, n}^{\prime} \\
\hline \varphi_{n-k+1,1}^{\prime} & \cdots & \varphi_{n-k+1, n-k}^{\prime} & \varphi_{n-k+1, n-k+1}^{\prime} & \cdots & \varphi_{n-k+1, n}^{\prime} \\
\cdots & \cdots & \cdots & \cdots & \cdots & \cdots \\
\hline \varphi_{n 1}^{\prime} & \cdots & \varphi_{n, n-k}^{\prime} & \varphi_{n, n-k+1}^{\prime} & \cdots & \varphi_{n n}^{\prime}
\end{array}\right]=\left[\begin{array}{ccc}
\Phi_{11}^{\prime} & \Phi_{12}^{\prime} & \Phi_{13}^{\prime} \\
\Phi_{21}^{\prime} & \Phi_{22}^{\prime} & \Phi_{23}^{\prime} \\
\Phi_{31}^{\prime} & \Phi_{32}^{\prime} & \Phi_{33}^{\prime}
\end{array}\right], \\
& \Gamma^{\prime}=F^{-1} \Gamma=\left[\begin{array}{c}
\Gamma_{1}^{\prime} \\
\Gamma_{2}^{\prime} \\
\Gamma_{3}^{\prime}
\end{array}\right], H^{\prime}=H F=\left[\begin{array}{lll}
H_{1}^{\prime} & H_{2}^{\prime} & H_{3}^{\prime}
\end{array}\right], x^{\prime}(t)=F^{-1} x(t)=\left[\begin{array}{c}
x_{1}^{\prime}(t) \\
\vdots \\
\vdots \\
x_{n}^{\prime}(t)
\end{array}\right],
\end{aligned}
$$

when $\varphi_{n, n-k+1}^{\prime} \neq 0$, the system (1), (2) can be changed into the following normal system

$$
\begin{aligned}
& x_{r}(t+1)=\Phi_{r} x_{r}(t)+\Gamma_{r} w(t), \\
& y(t)=H_{r} x_{r}(t)+v_{r}(t),
\end{aligned}
$$

where

$$
\begin{aligned}
& x_{r}(t)=\left[\begin{array}{c}
x_{1}^{\prime}(t) \\
\vdots \\
x_{n-k}^{\prime}(t) \\
x_{n-k+2}^{\prime}(t) \\
\vdots \\
x_{n}^{\prime}(t)
\end{array}\right], x_{n-k+1}^{\prime}(t)=-\frac{1}{\varphi_{n, n-k+1}^{\prime}}\left[\begin{array}{ll}
\Phi_{31}^{\prime} & \Phi_{33}^{\prime}
\end{array}\right] x_{r}(t)-\frac{1}{\varphi_{n, n-k+1}^{\prime}} \Gamma_{3}^{\prime} w(t) \\
& \Phi_{r}=\frac{1}{\varphi_{n, n-k+1}^{\prime}}\left[\begin{array}{cc}
J_{n-k}^{-1}\left(\varphi_{n, n-k+1}^{\prime} \Phi_{11}^{\prime}-\Phi_{12}^{\prime} \Phi_{31}^{\prime}\right) & J_{n-k}^{-1}\left(\varphi_{n, n-k+1}^{\prime} \Phi_{13}^{\prime}-\Phi_{12}^{\prime} \Phi_{33}^{\prime}\right) \\
\varphi_{n, n-k+1}^{\prime} \Phi_{21}^{\prime}-\Phi_{22}^{\prime} \Phi_{31}^{\prime} & \varphi_{n, n-k+1}^{\prime} \Phi_{23}^{\prime}-\Phi_{22}^{\prime} \Phi_{33}^{\prime}
\end{array}\right], \\
& \Gamma_{r}=\frac{1}{\varphi_{n, n-k+1}^{\prime}}\left[\begin{array}{c}
J_{n-k}^{-1}\left(\varphi_{n, n-k+1}^{\prime} \Gamma_{1}^{\prime}-\Phi_{12}^{\prime} \Gamma_{3}^{\prime}\right) \\
\varphi_{n, n-k+1}^{\prime} \Gamma_{2}^{\prime}-\Phi_{22}^{\prime} \Gamma_{3}^{\prime}
\end{array}\right], \\
& H_{r}=\frac{1}{\varphi_{n, n-k+1}^{\prime}}\left[\varphi_{n, n-k+1}^{\prime} H_{1}^{\prime}-H_{2}^{\prime} \Phi_{31}^{\prime} \quad \varphi_{n, n-k+1}^{\prime} H_{3}^{\prime}-H_{2}^{\prime} \Phi_{33}^{\prime}\right],
\end{aligned}
$$




$$
v_{r}(t)=v(t)-\frac{1}{\varphi_{n, n-k+1}^{\prime}} H_{2}^{\prime} \Gamma_{3}^{\prime} w(t),
$$

Proof. By the Jordan canonical matrix theory [6], if the matrix $M$ has $k$ zero eigenvalues, then there exists an invertible matrix $F$ such that $F^{-1} M F=M^{\prime}$. Let $x(t)=F x^{\prime}(t)$, substituting it into (1), (2) and premultiplying (1) by $F^{-1}$ yields (4), (5). If $\varphi_{n, n-k+1}^{\prime} \neq 0$ which is the last line of $\Phi^{\prime}$, we can obtain $x_{n-k+1}^{\prime}(t)=-\frac{1}{\varphi_{n, n-k+1}^{\prime}}\left[\begin{array}{ll}\Phi_{31}^{\prime} & \Phi_{33}^{\prime}\end{array}\right] x_{r}(t)-\frac{1}{\varphi_{n, n-k+1}^{\prime}} \Gamma_{3}^{\prime} w(t)$ by the last equation of (4). Substituting it into (4), (5) yields the normal system (9), (10).

\section{Descriptor Kalman filter}

Lemma 3 [7]. The discrete time stochastic system

$$
\begin{aligned}
& x(t+1)=\Phi x(t)+\Gamma w(t), \\
& y(t)=H x(t)+v(t),
\end{aligned}
$$

have the following Kalman filtering equations under Assumptions 2,3

$$
\begin{aligned}
& \hat{x}(t+1 \mid t+1)=\hat{x}(t+1 \mid t)+K(t+1) e(t+1), \\
& \hat{x}(t+1 \mid t)=\bar{\Phi} \hat{x}(t \mid t)+J y(t), \\
& e(t+1)=y(t+1)-H \hat{x}(t+1 \mid t), \\
& K(t+1)=P(t+1 \mid t) H^{\mathrm{T}}\left[H P(t+1 \mid t) H^{\mathrm{T}}+R\right]^{-1}, \\
& P(t+1 \mid t)=\bar{\Phi} P(t \mid t) \bar{\Phi}^{\mathrm{T}}+\Gamma\left(Q-S R^{-1} S^{\mathrm{T}}\right) \Gamma^{\mathrm{T}}, \\
& P(t+1 \mid t+1)=\left[I_{n}-K(t+1) H\right] P(t+1 \mid t), \\
& \hat{x}(0 \mid 0)=\mathrm{Ex}(0)=\mu_{0}, P(0 \mid 0)=E\left[\left(x(0)-\mu_{0}\right)\left(x(0)-\mu_{0}\right)^{\mathrm{T}}\right]=P_{0},
\end{aligned}
$$

where

$$
\bar{\Phi}=\Phi-J H, J=\Gamma S R^{-1} \text {. }
$$

From Lemma 1 and 2 we have

Theorem. The descriptor system (1), (2) have the following Kalman filtering equations under Assumptions 1 3

$$
\begin{aligned}
& \hat{x}_{r}(t+1 \mid t+1)=\hat{x}_{r}(t+1 \mid t)+K_{r}(t+1) e_{r}(t+1), \\
& \hat{x}_{r}(t+1 \mid t)=\bar{\Phi}_{r} \hat{x}_{r}(t \mid t)+J_{r} y(t), \\
& e_{r}(t+1)=y(t+1)-H_{r} \hat{x}_{r}(t+1 \mid t), \\
& K_{r}(t+1)=P_{r}(t+1 \mid t) H_{r}^{\mathrm{T}}\left[H_{r} P_{r}(t+1 \mid t) H_{r}^{\mathrm{T}}+R_{r}\right]^{-1}, \\
& P_{r}(t+1 \mid t)=\bar{\Phi}_{r} P_{r}(t \mid t) \bar{\Phi}_{r}^{\mathrm{T}}+\Gamma_{r}\left(Q-S_{r} R_{r}^{-1} S_{r}^{\mathrm{T}}\right) \Gamma_{r}^{\mathrm{T}},
\end{aligned}
$$




$$
\begin{aligned}
& P_{r}(t+1 \mid t+1)=\left[I_{n}-K_{r}(t+1) H_{r}\right] P_{r}(t+1 \mid t), \\
& \hat{x}_{r}(0 \mid 0)=\mathrm{E} x_{r}(0)=\mu_{r 0}, P_{r}(0 \mid 0)=E\left[\left(x_{r}(0)-\mu_{r 0}\right)\left(x_{r}(0)-\mu_{r 0}\right)^{\mathrm{T}}\right]=P_{r 0}, \\
& \hat{x}(t \mid t)=F \hat{x}^{\prime}(t \mid t)=F\left[\begin{array}{c}
\hat{x}_{1}^{\prime}(t \mid t) \\
\vdots \\
\vdots \\
\hat{x}_{n}^{\prime}(t \mid t)
\end{array}\right],
\end{aligned}
$$

where

$$
\begin{aligned}
& \hat{x}_{r}(t \mid t)=\left[\begin{array}{ll}
\hat{x}_{1}^{\prime}(t \mid t), \quad \cdots, \quad \hat{x}_{n-k}^{\prime}(t \mid t), \quad \hat{x}_{n-k+2}^{\prime}(t \mid t), \quad \cdots, \quad \hat{x}_{n}^{\prime}(t \mid t)
\end{array}\right]^{\mathrm{T}}, \\
& \hat{x}_{n-k+1}^{\prime}(t \mid t)=-\frac{1}{\varphi_{n, n-k+1}^{\prime}}\left[\Phi_{31}^{\prime} \quad \Phi_{33}^{\prime}\right] \hat{x}_{r}(t \mid t), \\
& \Phi_{r}=\Phi_{r}-J_{r} H_{r}, J_{r}=\Gamma_{r} S_{r} R_{r}^{-1}, \\
& R_{r}=R-\frac{1}{\varphi_{n, n-k+1}^{\prime}} S^{\mathrm{T}} \Gamma_{3}^{\prime \mathrm{T}} H_{2}^{\prime \mathrm{T}}-\frac{1}{\varphi_{n, n-k+1}^{\prime}} H_{2}^{\prime} \Gamma_{3}^{\prime} S+\frac{1}{\varphi_{n, n-k+1}^{\prime 2}} H_{2}^{\prime} \Gamma_{3}^{\prime} Q \Gamma_{3}^{\prime \mathrm{T}} H_{2}^{\prime \mathrm{T}}, \\
& S_{r}=S-\frac{1}{\varphi_{n, n-k+1}^{\prime}} Q \Gamma_{3}^{\prime \mathrm{T}} H_{2}^{\prime \mathrm{T}} .
\end{aligned}
$$

$\Phi_{r} 、 \Gamma_{r} 、 H_{r}$ is defined by (12) (14).

Proof. According to Lemma 2, the system (1), (2) can be changed into the normal system (9), (10). From Assumptions 2 and equation (15), we have

$w(t)$ and $v_{r}(t)$ are correlated white noises with zero mean, that is

$$
\mathrm{E}\left\{\left[\begin{array}{c}
w(t) \\
v_{r}(t)
\end{array}\right]\left[w^{\mathrm{T}}(j) \quad v_{r}^{\mathrm{T}}(j)\right]\right\}=\left[\begin{array}{cc}
Q & S_{r} \\
S_{r}^{\mathrm{T}} & R_{r}
\end{array}\right] \delta_{i j},
$$

where $R_{r}$ and $S_{r}$ are given by (37), (38). From Assumptions 3 and equation (15), we know that the initial state $x(0)$ is independent of $w(t)$ and $v_{r}(t)$. Then applying Lemma 3 yields (26) (32). According to the literature [8], we have $\hat{w}(t \mid t)=0$. Then equations (33) (35) can be obtained by (11). The theorem is proved.

\section{Conclusion}

In this paper, Jordan decomposition for matrices is used to change the descriptor system into the normal system in the first place, and then Kalman filtering theory for the normal system is used to introduce a new descriptor Kalman filter whose algorithm is recursive and simple, making it suitable for real-time application.

\section{Acknowledgments}

The research work was supported by the Foundation of Beijing Municipal Education Commission under Grant No. KM2014100150012 and Beijing high school youth talent plan (YETP1471). 


\section{References}

[1] R. Nikoukhah, A. S. Willsky and C. L. Bernard: IEEE Transactions on Automatica Control, Vol. 37 (1992) No.9, pp. 1325-1341.

[2] Z.L. Deng and Y. Xu: Control Theory and Applications, Vol. 16 (1999) No.5, pp.634-638. (In Chinese)

[3] Z.L. Deng and Y.M. Liu: International Journal of Systems Science, Vol. 30 (1999) No.11, pp. 1205-1212.

[4] H. S. Zhang, L. H. Xie and Y. C. Soh: IEEE Transactions on Automatic Control, Vol. 44 (1999) No.11, pp.2154-2158.

[5] L.S. Zeng: Journal of East China Shipbuilding Institute, Vol. 8 (1994) No.1, pp.101-105. (In Chinese)

[6] Y.P. Cheng: Matrix Theory (Xi'an: Northwestern Polytechnical University Press, China 2000).

[7] Z.L. Deng: Optimal Filtering Theory and Applications, Modern Time Series Analysis Method (Harbin: Harbin Institute of Technology Press, China 2000).

[8] Y. Xu and Z.L. Deng: Acta Automatica Sinica, Vol. 29 (2003) No.6, pp.835-841.

[9] Z.L. Deng and Y. Xu: Automatica, Vol. 36 (2000) No.11, pp.1761-1766. 